\title{
Can substitutions affect the oxidative stability of lithium argyrodite solid electrolytes?
}

\author{
Ananya Banik, ${ }^{a}$ Yunsheng Liu, ${ }^{b}$ Saneyuki Ohno, ${ }^{c}$ Yannik Rudel, ${ }^{a}$ Alberto Jiménez-Solano, ${ }^{d}$ \\ Andrei Gloskovskii, ${ }^{e}$ Nella M. Vargas-Barbosa, ${ }^{f}$ Yifei Mo, ${ }^{b *}$ and Wolfgang G. Zeier ${ }^{a, f *}$ \\ ${ }^{a}$ Institute of Inorganic and Analytical Chemistry, University of Münster, Corrensstrasse 30, 48149 \\ Münster, Germany. \\ ${ }^{b}$ Department of Materials Science and Engineering, University of Maryland, College Park, Maryland \\ 20742, United States. \\ ${ }^{c}$ Department of Applied Chemistry, Graduate School of Engineering, Kyushu University, 744 \\ Motooka, Nishi-ku, 819-0395 Fukuoka, Japan. \\ ${ }^{d}$ Max Planck Institute for Solid State Research, Heisenbergstraße 1, 70569 Stuttgart, Germany. \\ ${ }^{e}$ Deutsches Elektronen-Synchrotron DESY 22607, Hamburg, Germany. \\ ${ }^{f}$ Institut für Energie- und Klimaforschung (IEK), IEK-12: Helmholtz-Institut Münster, \\ Forschungszentrum Jülich, Corrensstrasse 46, 48149 Münster, Germany. \\ Corresponding authors*: yfmo@umd.edu,wzeier@uni-muenster.de
}

Lithium ion conducting argyrodites are among the most studied solid electrolytes due to their high ionic conductivities. A major concern in a solid-state battery is the solid electrolyte stability. Here we present a systematic study on the influence of cationic and anionic substitution on the electrochemical stability of $\mathrm{Li}_{6} \mathrm{PS}_{5} X$, using step-wise cyclic voltammetry, optical band gap measurements, hard X-ray photoelectron spectroscopy along with first-principles calculations. We observe that going from $\mathrm{Li}_{6} \mathrm{PS}_{5} \mathrm{Cl}$ to $\mathrm{Li}_{6+\mathrm{x}} \mathrm{P}_{1-\mathrm{x}} M_{\mathrm{x}} \mathrm{S}_{5} \mathrm{I}\left(M=\mathrm{Si}^{4+}, \mathrm{Ge}^{4+}\right)$, the oxidative degradation does not change. Considering the chemical bonding shows that the valence band edges are mostly populated by non-bonding orbitals of the $\mathrm{PS}_{4}{ }^{3-}$ units or unbound sulfide anions and that simple substitutions in these sulfide-based solid electrolytes cannot improve oxidative stabilities. This work provides insights on the role of chemical bonding on the stability of superionic conductors and shows that alternative strategies are needed for long-term stable solid-state batteries.

\section{TOC Graphic}

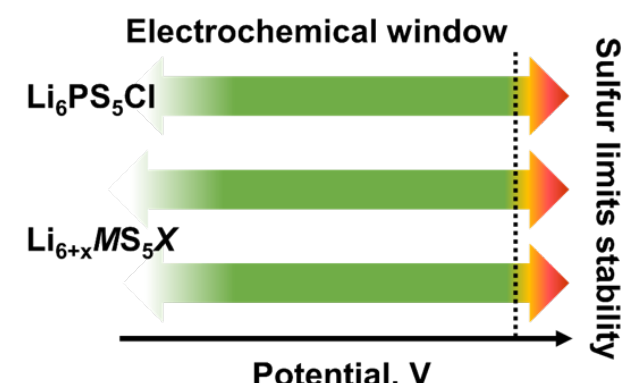


The all-solid-state battery has garnered interest as a viable alternative to conventional Li-ion batteries. ${ }^{1,2}$ Successful production of a solid-state battery requires a solid electrolyte (SE) with high Li-ion conductivity and wide (electro)chemical stability window. ${ }^{3-5}$ The lithium-ion conducting thiophosphates are currently used for solid electrolytes due to their high ionic conductivity and mechanical softness. ${ }^{6-9}$ Recent efforts have led to the discovery of various thiophosphate electrolytes such as $\mathrm{Li}_{6} \mathrm{PS}_{5} X(X=\mathrm{Cl}, \mathrm{Br}, \mathrm{I}),{ }^{6,10-13} \mathrm{Li}_{10} M \mathrm{P}_{2} \mathrm{~S}_{12}(M=\mathrm{Ge}, \mathrm{Sn}),{ }^{9,14,15}$ and $\mathrm{Li}_{2} \mathrm{~S}-\mathrm{P}_{2} \mathrm{~S}_{5}$ glass-ceramic phases ${ }^{16}$. Nevertheless, the main concern related to the use of these solid electrolytes is a limited understanding of the solid electrolyte - electrode interfacial (in)stability. ${ }^{17-19}$ In most cases, the solid electrolyte reacts with electrode materials leading to the formation of passivating interphase layers, similar to what is observed with liquid electrolytes in Li-ion batteries. ${ }^{19-22}$ Even though an interphase layer may potentially stop further solid electrolyte degradation and thus enable long-term cycling, solid-state battery performance would be poorer due to the increased cell resistance. Therefore, two routes have emerged to promote long-term cycling that focus either on incorporating protective coatings in active materials ${ }^{19,23}$ or utilizing compositional changes in known solid electrolyte phases to achieve higher stabilities ${ }^{24-26}$. While the former option has shown to be very effective, it is unclear if substitution in sulfide solid electrolytes can enhance their stability towards the electrode materials for operation. Further, recent reports have explored the role of electronically insulating additives to improve electrochemical stability of thiophosphates, ${ }^{27}$ however the reason behind enhanced stability is not clear yet.

In principle, there are multiple approaches to determine the electrochemical stability window of a solid electrolyte. The stability window is defined as the voltage range in which the electrolyte does not participate in charge-transfer reactions with the electrode materials. In other words, the electrolyte is neither reduced nor oxidized at the electrolyte-electrode interface. The three possible approaches to determine the electrochemical stability windows are (1) the "band edge approach", (2) the "stoichiometry stability approach" and (3) the "phase stability approach". All of these are in-depth described and compared to each other by Binninger et al. ${ }^{28}$ The band edge approach (or sometimes called HOMO-LUMO) suggests that to prevent the reduction of a solid electrolyte at the anode, the Fermi level of the anode $\left(\varepsilon_{F}\right.$,anode $)$ should be at lower energies than the conduction band minimum of the solid electrolyte. Similarly, to prevent oxidative decomposition, the electrochemical potential of the cathode

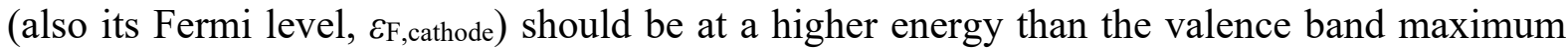
shown schematically in Figure $1 \mathrm{a} .{ }^{29}$ The energy difference between the conduction band 
minimum and the valence band maximum is the band gap. This is the reason for the more often used terminology of "band gap approach". Whereas the "stoichiometry stability approach" is mostly used for insertion/extraction reactions, the "phase stability approach" is used to calculate intrinsic thermodynamic stability windows of solid electrolytes..$^{20,21,28,30}$ The "phase stability approach" considers the electrolyte as a reactant that can be fully oxidized or reduced to produce specific products at specific (redox) potentials. These decomposition reactions are directly related to the Gibbs free energies of the redox reactions(s). ${ }^{28,31}$ In short, the "band edge approach" is expected to determine which element drives the decomposition and provide an upper limit to the stability window, while the thermodynamic electrochemical stability approach defines the decomposition potential(s) of a solid electrolyte (see Figure 1b). However, it is often observed that the stability windows theoretically predicted tend to be much narrower than those observed experimentally (Figure 1c). ${ }^{17,18,32,33}$ The mismatch between theory and experiment is due to the fact that interfacial reaction kinetics and intermediate metastable phases are not fully accounted for in the calculations. ${ }^{17,34,35}$ Additionally, different measurement approaches for the stability window can limit comparability. Recent attempts to better determine the experimental electrochemical stability window have used carbon - solid electrolyte composites and then either stepwise cyclic voltammetry ${ }^{17,18,36}$ or linear sweep voltammetry ${ }^{37}$ to measure these electrochemical stabilities. ${ }^{37}$
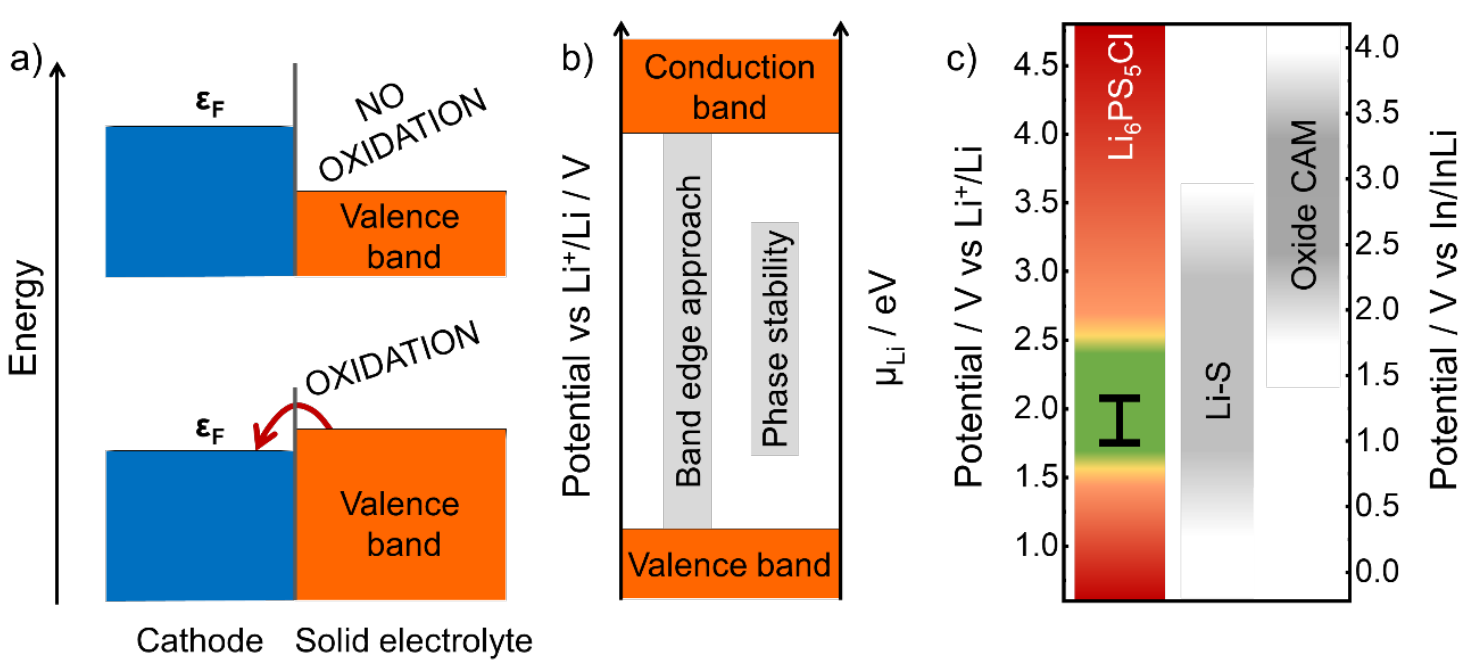

Figure 1. a) Schematic of oxidative degradation mechanism of a solid electrolyte in contact with a cathode active material based on band theory of solids. Oxidative degradation of the solid electrolyte involves electron transfer from the valence band edge to the cathode. Thus, for a stable electrolyte, the valence band minimum needs to be at energies below the Fermi level $\varepsilon_{F}$ of cathode. The atoms that contribute to the band edges participate in the electron 
transfer process with cathode during decomposition. b) Comparison of typical electrochemical stability window obtained from band gap approach and phase stability method. c) Comparison of practical stability window of $\mathrm{Li}_{6} \mathrm{PS}_{5} \mathrm{Cl}$ (green colored region) with thermodynamic phase stability (marked by double-headed arrow). Oxidative and reductive decomposition of $\mathrm{Li}_{6} \mathrm{PS}_{5} \mathrm{Cl}$ is closely related to the sulfur and phosphorous redox potentials. Oxidative decomposition starts at $2.5 \mathrm{~V} v \mathrm{In} / \mathrm{InLi}$, whereas reductive decomposition occurs below $0.6 \mathrm{~V}$ vs In/InLi. ${ }^{17}$ The calculated thermodynamic phase stability window ${ }^{21}$ is narrower than the measured one. Typical cycling windows of $\mathrm{Li}-\mathrm{S}$ and oxide cathode active materials (CAM) are also shown for comparison.

Clearly, solid electrolytes must have an upper bound to their oxidative stability. For instance, in the case of $\mathrm{Li}_{6} \mathrm{PS}_{5} \mathrm{Cl}$, its oxidative stability is within the operating voltage range of sulfur as a cathode material, but outside of the typical range of oxide-based insertion cathode active materials (Figure 1c). ${ }^{17,18}$ It is therefore critical to elucidate if these argyrodites can be improved to achieve a higher oxidative stability. This question is especially important since tailoring the composition is a typical approach to improve ionic conductivities, ${ }^{5}$ yet it is unclear if the stability is also affected.

In order to answer the question if altering the composition affects the oxidative electrochemical degradation, in this work we compare the electrochemical stability of sulfide based argyrodites $\mathrm{Li}_{6+}{ }_{\mathrm{x}} M \mathrm{~S}_{5} X$ by changing the composition $\left(M=\mathrm{P}^{5+}, \mathrm{Si}^{4+}, \mathrm{Ge}^{4+} ; X=\mathrm{Cl}^{-}, \mathrm{I}^{-}\right)$. Using a combination of hard X-ray photoemission spectroscopy to unravel the band edges together with optical band gap measurements, we compare these experimental data to theoretical calculations of the band structure and the phase stability. Additionally, step-wise cyclic voltammetry is used to measure the changes in the practical oxidative stability. Here, we show that changing the composition in these argyrodite-based ionic conductors barely affect the thermodynamic and electrochemical stability. By understanding the chemical nature of the band edges, we show that as long as sulfur is part of the solid electrolyte, the oxidative stability will always be limited, irrespective of the full chemical composition.

Lithium-ion conducting halide argyrodites, $\mathrm{Li}_{6} \mathrm{PS}_{5} X$ have been of particular interest because of their high ionic conductivity, their negligible grain boundary resistance, and the possibility to change the ionic conductivity via substitutions. The oxidative decomposition pathway of $\mathrm{Li}_{6} \mathrm{PS}_{5} \mathrm{Cl}$ has been explored in depth, ${ }^{17,18}$ and the underlying chemical reactions occur due to the sulfur being readily oxidized. $\mathrm{Li}_{6} \mathrm{PS}_{5} \mathrm{Cl}$ consists of $\mathrm{PS}_{4}{ }^{3-}$ ortho-thiophosphate species, 
together with $\mathrm{S}^{2-}$ and $\mathrm{Cl}^{-}$anions. At the stability limit, the oxidative degradation involves the oxidation of $\mathrm{PS}_{4}{ }^{3-}$ and $\mathrm{S}^{2-}$ anions, forming polysulfides, sulfur and additional (P-S $)_{\mathrm{x}}$ units. ${ }^{18,19}$ After decomposition, these units themselves become redox active. ${ }^{18}$

In order to understand how compositional substitutions change the oxidative stability of these argyrodites, we employ $\mathrm{Li}_{6} \mathrm{PS}_{5} \mathrm{Cl}$ as the baseline material and use substituted-Li ${ }_{6} \mathrm{PS}_{5} \mathrm{I}$, $\mathrm{Li}_{6.6} \mathrm{P}_{0.4} \mathrm{Si}_{0.6} \mathrm{~S}_{5} \mathrm{I}$ and $\mathrm{Li}_{6.6} \mathrm{P}_{0.4} \mathrm{Ge}_{0.6} \mathrm{~S}_{5} \mathrm{I}$ as additional model systems. We aim to elucidate how the oxidative stability is affected in argyrodites by employing cationic substitution, replacing $\mathrm{P}$ with $\mathrm{Si}$ or $\mathrm{Ge}$, and anionic substitution, exchanging $\mathrm{Cl}$ with I. The Ge and Si substituted model systems have been chosen due to their similar ionic conductivities, ${ }^{6,8,38}$ which allow for the experimental measurement of the electrochemical stability and excludes mass-transport effects as an influencing factor. Unfortunately, the low ionic conductivity of unsubstituted $\mathrm{Li}_{6} \mathrm{PS}_{5} \mathrm{I}$ excludes it from electrochemical measurements, ${ }^{39}$ nevertheless for the theoretical work it was used as an additional benchmark to compare the direct change from $\mathrm{Li}_{6} \mathrm{PS}_{5} \mathrm{Cl}$ to $\mathrm{Li}_{6} \mathrm{PS}_{5} \mathrm{I}$.

Practical electrochemical stability. To understand the influence of the composition on the electrochemical stability, we determine the stability limit using a step-wise cyclic voltammogram approach, that has recently been shown to be effective for elucidating the stability of sulfide solid electrolytes. ${ }^{17,18}$ Since the oxidative stability of $\mathrm{Li}_{6} \mathrm{PS}_{5} X$ is the major concern for its usage in a solid-state battery, here we focus on the oxidative degradation only. Carbon - solid electrolyte composites are used to enhance the interfacial areas. The voltammogram is recorded twice up to this reversal potential followed by a stepwise increase of the potential range by $0.1 \mathrm{~V}$ up to $3 \mathrm{~V}$ vs. In/InLi (Figure 2 and Figure S3). As seen in Figure 2a-c, below a certain voltage (1.8 V vs In/InLi), only a capacitive current can be observed. After that, with increasing voltage, a significant increase in current was observed. The peak current of each scan rises drastically when increasing the reversal voltage above $1.8 \mathrm{~V}$ vs In/InLi. Additionally, after the decomposition, anodic and cathodic features start to evolve indicating the known redox-activity of the decomposition products. ${ }^{18}$ Typically, for $\mathrm{Li}_{6} \mathrm{PS}_{5} \mathrm{Cl}$,

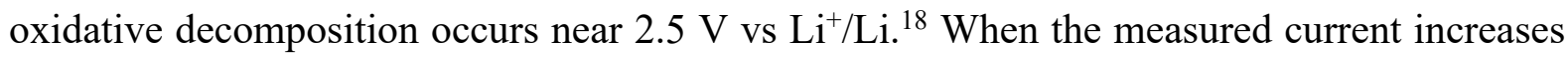
as a function of the potential (Figure 2d), all systems show similar onsets of decomposition by increasing currents. In other words, the oxidative stability window does not change abruptly from $\mathrm{Li}_{6} \mathrm{PS}_{5} \mathrm{Cl}$ to substituted $\mathrm{Li}_{6} \mathrm{PS}_{5} \mathrm{I}$ indicating that neither the anion nor the cation substitutions affect the practical oxidative electrochemical stability. 

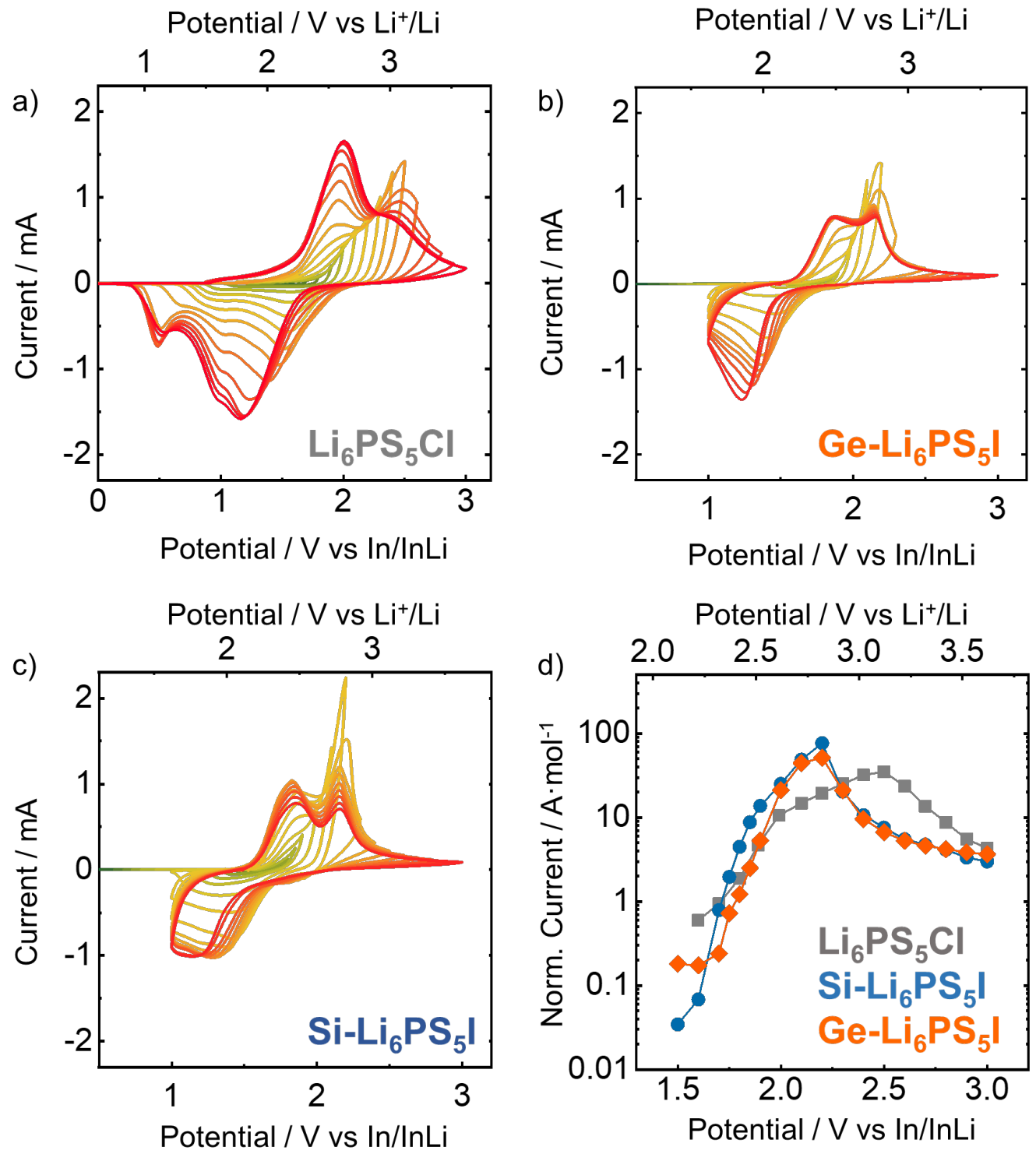

Figure 2. Stepwise cyclic voltammogram for $\mathrm{Li}_{6} P \mathrm{PS}_{5} \mathrm{Cl}$ and $\mathrm{Ge}-\mathrm{Li}_{6} \mathrm{PS}_{5} \mathrm{I}$ and Si--Li $\mathrm{i}_{6} \mathrm{PS} I$ I $(a, b$ and c). Oxidative decomposition at high potentials leads to evolving peaks with varying peak area at potentials at which the electrolyte was apparently stable when lower reversal potentials were applied. Open-circuit voltage was $0.5 \mathrm{~V} v \mathrm{~s}$. In/InLi. d) Comparison of oxidative currents at reversal potentials of $C V$ scans normalized by moles of material to decouple from potential compositional differences.

Measuring band edges and calculating stability windows. Although there are a few reports on the understanding of electronic structure and how it influences the (electro)chemical stability, there are no experimental studies focusing on the chemical nature of the band edges and their relation to the electrochemical decomposition of solid electrolytes. We have measured the optical band gap and valence band spectra to shed light on the electronic structure of these 
materials (Figure $3 \mathrm{a}$ and $\mathrm{b}$ ). All the materials exhibit direct band gap values with above $3 \mathrm{eV}$, similar to the ones observed theoretically (Figure $3 \mathrm{a}$ and Table S1). Hard X-ray photoemission spectroscopy (HAXPES) is a common tool to visualize the valence band edge of bulk material. ${ }^{40}$ While it is primarily used in semiconductors with high electronic conductivities, its usage is rare in studies of ionic conductors. To avoid the surface contribution, we have used Xray with high energy $(6 \mathrm{keV})$ which is a typical energy source used to probe bulk materials. ${ }^{41}$ Unfortunately, no HAXPES data collection was possible for $\mathrm{Li}_{6} \mathrm{PS}_{5} \mathrm{I}$, due to strong sample changing. Figure $3 \mathrm{~b}$ shows the valence band photoelectron spectra for $\operatorname{Li}_{6+{ }_{x}} \mathrm{P}_{1-\mathrm{x}} M_{\mathrm{x}} \mathrm{S}_{5} \mathrm{X}$. The HAXPES features correspond to electronic density of occupied states near Fermi level. According to Figure $3 b$, for all the materials, the first peak appears at a similar energy, suggesting similar valence band edge energies of these electronic insulators. To understand the electronic structure and its impact on redox activity, we have calculated the electronic structure using density functional theory. We have chosen the compositions of $\operatorname{Li}_{6.25} \mathrm{P}_{0.75} \mathrm{Si}_{0.25} \mathrm{~S}_{5} \mathrm{I}$ and $\mathrm{Li}_{6.75} \mathrm{P}_{0.25} \mathrm{Ge}_{0.75} \mathrm{~S}_{5} \mathrm{I}$ as $\mathrm{Si}$ - and Ge- substituted $\mathrm{Li}_{6} \mathrm{PS}_{5} \mathrm{I}$ for theoretical calculation since these are the nearest to the actual composition of experimentally prepared compounds. ${ }^{6,8}$ Both valence band maxima and conduction band minima occur at the $\Gamma$-point, confirming direct band gap, as seen in Figure S4. From the electronic structure calculations, diffuse reflectance spectra and HAXPES measurements it is clear that the valence band maxima do not change significantly upon substitution. Slight shifts in the band gap are observed which can hence be associated with the change in the conduction band position upon cationic substitution.

To understand the thermodynamics of the electrochemical degradation pathway, we have calculated the lithium evolution number as a function of potential (Figure S5). From these calculations we obtain the thermodynamic stability window shown in Figure 3c, with the thermodynamic decomposition products listed in Table S2. In direct comparison, the here determined practical electrochemical stability windows (Figure 3d) closely resemble the calculated stability window, albeit a slightly larger stability. Unlike previous reports, ${ }^{18}$ the reaction kinetics were enhanced with the use of a highly conducting higher surface area carbon, leading to a better comparison to experiment. Interestingly, $\mathrm{Li}_{6} \mathrm{PS}_{5} \mathrm{I}$ seems to exhibit a slightly higher thermodynamic stability than the substituted ones, making $\mathrm{Li}_{6} \mathrm{PS}_{5} \mathrm{I}$ an intermediate degradation product for the decomposition of $\mathrm{Ge}_{-} \mathrm{Li}_{6} \mathrm{PS}_{5} \mathrm{I}$. From the thermodynamic decomposition products it is clear that decomposition of these sulfide solid electrolyte leads to formation of electrochemically active compounds such as sulfur, leading to the observed redox 
behavior as shown in Figure $2 .{ }^{18}$ Besides, LiX is found as one of the decomposition products, which has been recently proved to enhance the oxidative stability of thiophosphate, $\mathrm{Li}_{3} \mathrm{PS}_{4}{ }^{27}$
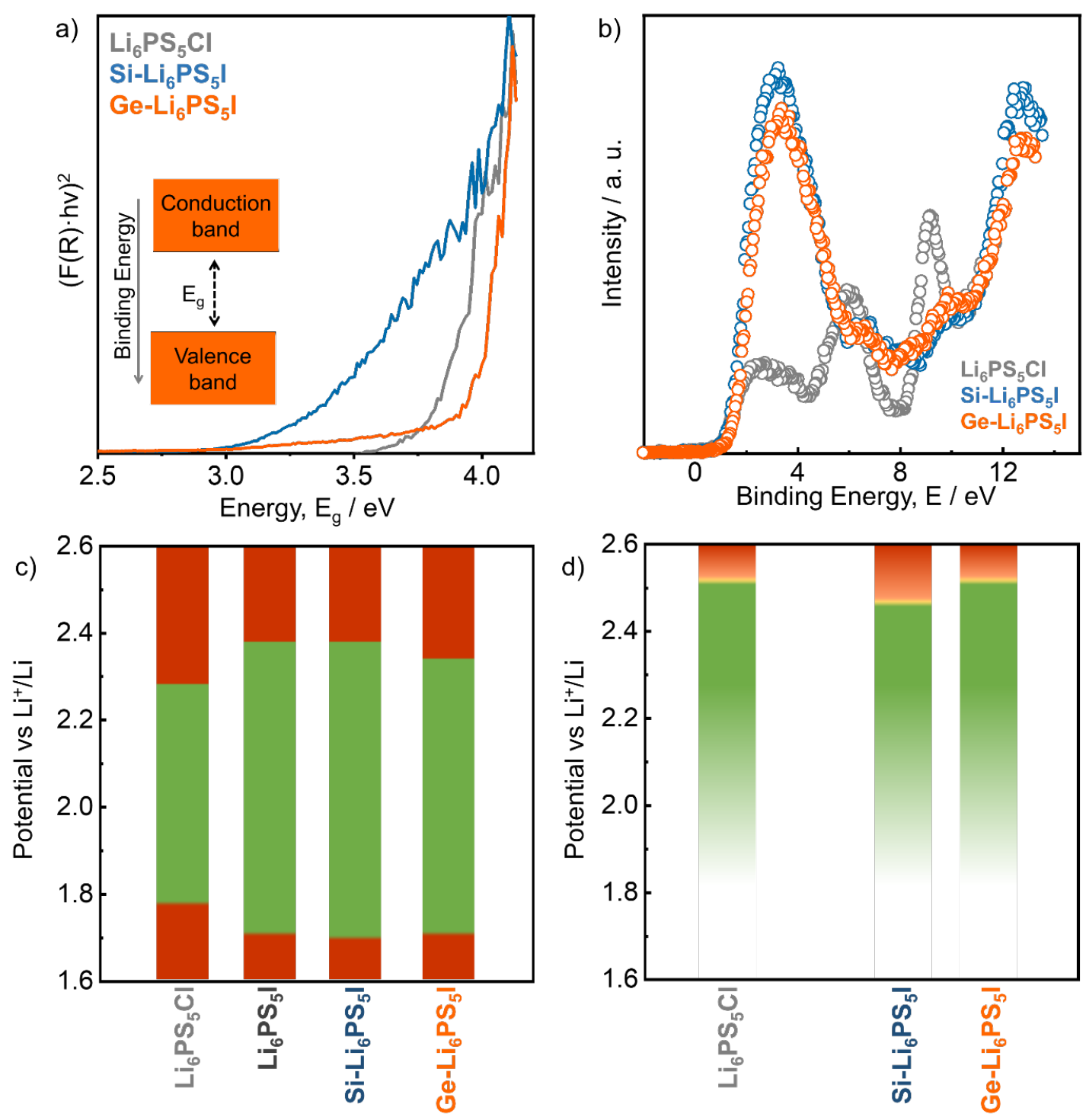

Figure 3. a) Optical absorption spectra and b) hard X-ray photoemission spectra showing band gap, $E_{g}$ and valence band edges, respectively. c) Calculated thermodynamic electrochemical stability windows and d) experimental oxidative stability limits. The stable region for each electrolyte is shown in green and the onset of oxidative decomposition is shown in orange followed by red to show the unstable potential region.

Clearly the substitutions via the halide anion or substitution of the cation in $\mathrm{PS}_{4}{ }^{3-}$ units do not significantly affect the stability of these sulfide argyrodites solid ionic conductors. To shed light on the relation between electronic structure and electrochemical stability, we take a closer 
look at the contributions of all atoms to the band edges. Partial density of states (pDOS) as well as crystal orbital Hamilton populations (COHP) were calculated for all the compounds (Figure 4 and Figure S6). These contributions can be qualitatively understood considering a simplified schematic of pDOS (Figure 4a) considering the valence state energy states based the electronegativity of the atoms and with it their atomic orbital energies. Whereas the pDOS give an idea of the atomic contributions to the different bands and electronic states, the COHP provide information about which states exhibit bonding or anti-bonding chemical interactions.

Considering the pDOS, in all the investigated lithium argyrodites the valence bands are mostly composed of anion states, of which sulfur seems to dominate the valence band edge. In the case of $\mathrm{Li}_{6} \mathrm{PS}_{5} \mathrm{Cl}$ the $\mathrm{Cl}$-states are located deeper in the valence band, whereas for the I-based materials the halide states can also be found at the valence band edge (Figure S6). However, according to the thermodynamic phase equilibria calculation, $\mathrm{I}^{-} / \mathrm{I}_{2}$ redox does not influence the electrochemical stability window of argyrodite solid electrolytes (Table S3) and can be neglected. It is noteworthy that this stronger influence of the halide at the band edge seems to be reflected in the HAXPES data in which the I-based materials exhibit a stronger intensity at the band edge (see Figure $3 \mathrm{~b}$ and Figure S6). Nevertheless, sulfur states dominate the valence band edge, irrespective of the halide composition. In the conduction band, the cationic contributions, especially those from $\mathrm{P}$, are stronger leading to nearly equal contributions of $\mathrm{S}$, suggesting an even stronger influence of $\mathrm{PS}_{4}{ }^{3-}$ on the conduction band edge. This is corroborated by the COHP which highlights the contributions of $\mathrm{PS}_{4}{ }^{3-}$ units on the electronic structure. The conduction band edge is populated mainly with P-S antibonding states while the P-S bonding states lie far below the valence band edge. Since the P-S bonding states of the $\mathrm{PS}_{4}{ }^{3-}$ units are so low in energy, this means that the filled non-bonding sulfur orbitals express as heavy states in the electronic structure and form the valence band edge for these materials. In $\mathrm{Li}_{6} \mathrm{PS}_{5} \mathrm{Cl}$, the higher electronegativity of chlorine shifts the $3 p$ states below the valence band edge. For the iodide materials, even though both the free sulfur and iodine contributes to the valence band edge, the major contribution comes from non-bonding $p$-states of the sulfur atoms of $\mathrm{PS}_{4}{ }^{3-}$ unit and the $\mathrm{S}^{2-}$ anion in the structure. These bonding considerations show that even if substitutions are performed on the $\mathrm{MS}_{4}$ units or the halide composition, the energy state of the valence band maximum is mostly determined by the sulfur. Substitutions can only affect the magnitude of the band gaps as stronger bonding interactions in the $\mathrm{MS}_{4}$ units would then shift the conduction band minimum to higher energies, as reflected in the measured optical band gaps. 

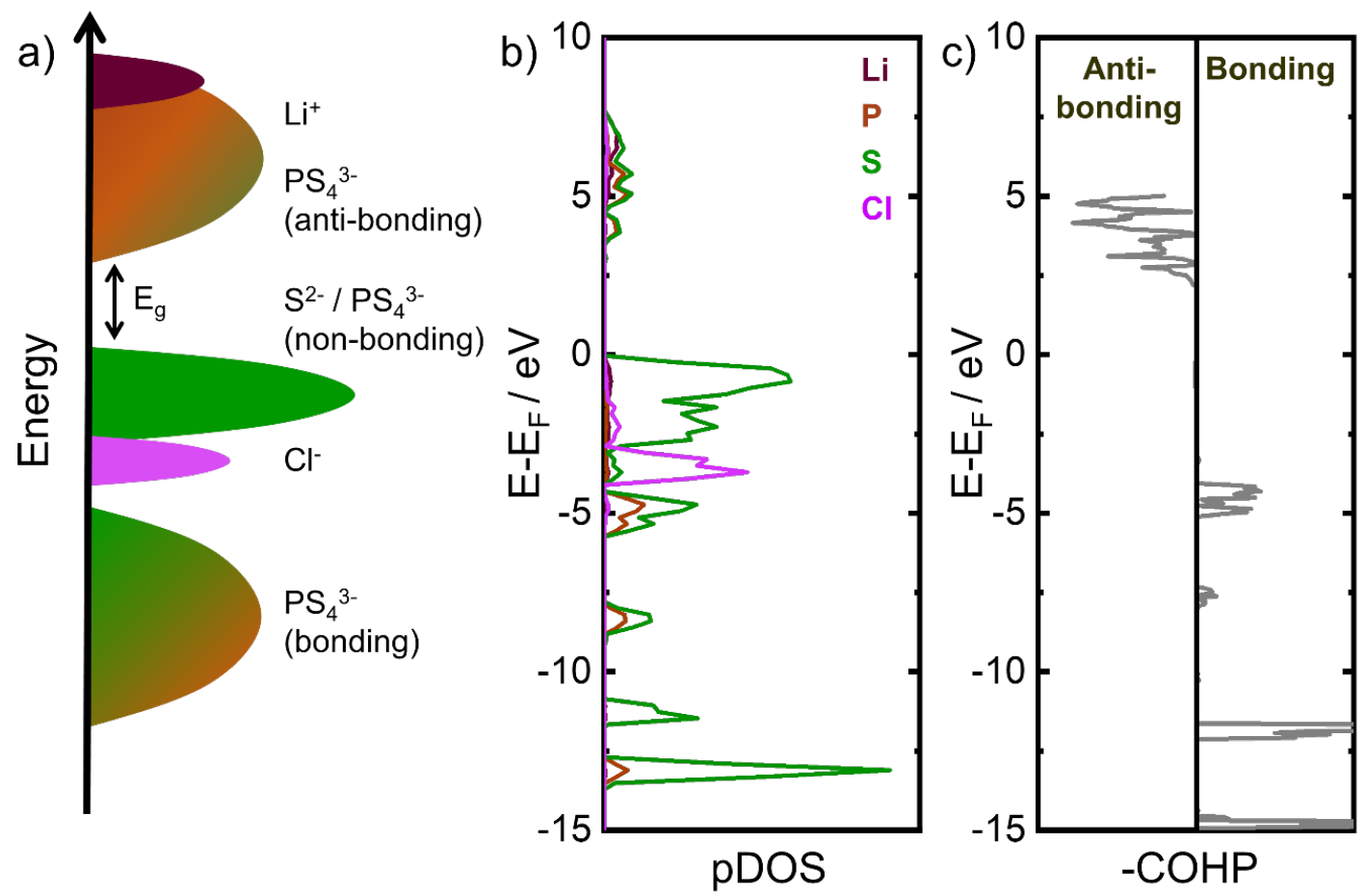

Figure 4. Electronic structure of $\mathrm{Li}_{6} \mathrm{PS}_{5} \mathrm{Cl}$. a) Schematic of pDOS showing major contribution of free $\mathrm{S}^{2-}$ and non-bonding sulfur states from the $\mathrm{PS}_{4}{ }^{3-}$ unit in the valence band edge, whereas the conduction band is dominated by antibonding states of $\mathrm{PS}_{4}^{3-}$. Because of a larger electronegativity, the $\mathrm{Cl}^{-}$states reside deeper in the valence and do not participate in bonding with phosphorous. The $\mathrm{PS}_{4}{ }^{3-}$ bonding states are far below the valence band maximum. b) Partial, atom-projected DOS shows the $\mathrm{PS}_{4}{ }^{3-}$ unit along with free $\mathrm{S}^{2-}$ and $\mathrm{Cl}^{-}$make up the valence band. c) Crystal Orbital Hamilton populations (COHP) shows the bonding antibonding contributions of $\mathrm{PS}_{4}{ }^{3-}$ in the electronic structure.

These electronic structure considerations of the argyrodites show why substitutions in argyrodite-based materials are hardly affecting the oxidative stability of the materials. Sulfur states dominate the band edge, effectively pinning the oxidative stability window. Thus, one can expect a similar oxidative stability for all sulfide solid electrolytes, regardless of the full composition. As the exact thermodynamic stability is linked to the decomposition pathways, minor changes in oxidative stability are expected, however, the driving force for the decomposition remains at the sulfur band edges. 
In summary, we use a combination of electronic structure calculations, thermodynamic phase stability calculations along with experimental determination of band edges and effective oxidative stability windows in the argyrodite family of superionic conductors. By elucidating the chemical nature of the band edges and developing an in-depth understanding of the bonding interactions, we demonstrate sulfur to be the Achilles' heel of the oxidative stability of sulfide solid electrolytes. Our results show that simple substitutions in sulfide solid electrolytes can barely change their intrinsic oxidative electrochemical stability, and with it the decomposition pathways, if sulfur remains part of the chemical composition. Therefore, for long-term stable operation of solid-state batteries, cathode active material coatings or different materials classes are needed altogether.

\section{Additional Information}

\section{Notes}

The authors declare no conflict of interest.

\section{ACKNOWLEDGMENTS}

The research was supported by the Federal Ministry of Education and Research (BMBF) within project FESTBATT under grant number 03XP0430F. This research used resources at the P22 beamline at the light source PETRA III of DESY, a member of the Helmholtz Association (HGF). A. B. and S. O. gratefully acknowledge the Alexander von Humboldt Foundation for financial support through a Postdoctoral Fellowship. Y.L and Y.M acknowledge the funding support from National Science Foundation HDR Program under award number 1940166, and the computational facilities from the University of Maryland supercomputing resources and the Maryland Advanced Research Computing Center (MARCC).

\section{SUPPORTING INFORMATION}

Laboratory X-ray diffraction data along with Pawley fit; room temperature impedance spectra; comparison of maximum currents at reversal potential during $\mathrm{CV}$; electronic structures; Lithium-evolution number vs voltage plot; pDOS and COHP; band gap; phase equilibria at the reduction and oxidation potentials of the solid electrolyte; phase equilibria at different applied potentials 


\section{References}

(1) Janek, J.; Zeier, W. G. A Solid Future for Battery Development. Nat. Energy 2016, 1 (9), 16141. https://doi.org/10.1038/nenergy.2016.141.

(2) Randau, S.; Weber, D. A.; Kötz, O.; Koerver, R.; Braun, P.; Weber, A.; Ivers-Tiffée, E.; Adermann, T.; Kulisch, J.; Zeier, W. G.; Richter, F. H.; Janek, J. Benchmarking the Performance of All-Solid-State Lithium Batteries. Nat. Energy 2020, 5 (3), 259-270. https://doi.org/10.1038/s41560-020-0565-1.

(3) Wang, Y.; Richards, W. D.; Ong, S. P.; Miara, L. J.; Kim, J. C.; Mo, Y.; Ceder, G. Design Principles for Solid-State Lithium Superionic Conductors. Nat. Mater. 2015, 14 (10), 1026-1031. https://doi.org/10.1038/nmat4369.

(4) Culver, S. P.; Koerver, R.; Krauskopf, T.; Zeier, W. G. Designing Ionic Conductors: The Interplay between Structural Phenomena and Interfaces in Thiophosphate-Based Solid-State Batteries. Chem. Mater. 2018, 30 (13), 4179-4192. https://doi.org/10.1021/acs.chemmater.8b01293.

(5) Ohno, S.; Banik, A.; Dewald, G. F.; Kraft, M. A.; Krauskopf, T.; Minafra, N.; Till, P.; Weiss, M.; Zeier, W. G. Materials Design of Ionic Conductors for Solid State Batteries. Prog. Energy 2020, 2 (2), 022001. https://doi.org/10.1088/25161083/ab73dd.

(6) Kraft, M. A.; Ohno, S.; Zinkevich, T.; Koerver, R.; Culver, S. P.; Fuchs, T.; Senyshyn, A.; Indris, S.; Morgan, B. J.; Zeier, W. G. Inducing High Ionic Conductivity in the Lithium Superionic Argyrodites Li6+xP1-XGexS5I for All-Solid-State Batteries. $J$. Am. Chem. Soc. 2018, 140 (47), 16330-16339. https://doi.org/10.1021/jacs.8b10282.

(7) Kraft, M. A.; Culver, S. P.; Calderon, M.; Böcher, F.; Krauskopf, T.; Senyshyn, A.; Dietrich, C.; Zevalkink, A.; Janek, J.; Zeier, W. G. Influence of Lattice Polarizability on the Ionic Conductivity in the Lithium Superionic Argyrodites Li6PS5X (X $=\mathrm{Cl}, \mathrm{Br}$, I). J. Am. Chem. Soc. 2017, 139 (31), 10909-10918.

https://doi.org/10.1021/jacs.7b06327.

(8) Ohno, S.; Helm, B.; Fuchs, T.; Dewald, G.; Kraft, M. A.; Culver, S. P.; Senyshyn, A.; Zeier, W. G. Further Evidence for Energy Landscape Flattening in the Superionic Argyrodites Li6+ XP1- XMxS5I (M = Si, Ge, Sn). Chem. Mater. 2019, 31 (13), 4936- 
4944. https://doi.org/10.1021/acs.chemmater.9b01857.

(9) Kamaya, N.; Homma, K.; Yamakawa, Y.; Hirayama, M.; Kanno, R.; Yonemura, M.; Kamiyama, T.; Kato, Y.; Hama, S.; Kawamoto, K.; Mitsui, A. A Lithium Superionic Conductor. Nat. Mater. 2011, 10 (9), 682-686. https://doi.org/10.1038/nmat3066.

(10) Adeli, P.; Bazak, J. D.; Park, K. H.; Kochetkov, I.; Huq, A.; Goward, G. R.; Nazar, L. F. Boosting Solid-State Diffusivity and Conductivity in Lithium Superionic Argyrodites by Halide Substitution. Angew. Chemie - Int. Ed. 2019, 58 (26), 86818686. https://doi.org/10.1002/anie.201814222.

(11) Gautam, A.; Sadowski, M.; Prinz, N.; Eickhoff, H.; Minafra, N.; Ghidiu, M.; Culver, S. P.; Albe, K.; Fässler, T. F.; Zobel, M.; Zeier, W. G. Rapid Crystallization and Kinetic Freezing of Site-Disorder in the Lithium Superionic Argyrodite Li6PS5Br. Chem. Mater. 2019, 31 (24), 10178-10185. https://doi.org/10.1021/acs.chemmater.9b03852.

(12) Zhou, L.; Minafra, N.; Zeier, W. G.; Nazar, L. F. Innovative Approaches to LiArgyrodite Solid Electrolytes for All-Solid-State Lithium Batteries. Acc. Chem. Res. 2021, 54 (12), 2717-2728. https://doi.org/10.1021/acs.accounts.0c00874.

(13) Zhou, L.; Assoud, A.; Zhang, Q.; Wu, X.; Nazar, L. F. New Family of Argyrodite Thioantimonate Lithium Superionic Conductors. J. Am. Chem. Soc. 2019, 141 (48), 19002-19013. https://doi.org/10.1021/jacs.9b08357.

(14) Krauskopf, T.; Culver, S. P.; Zeier, W. G. Bottleneck of Diffusion and Inductive Effects in Li10Ge1- XSnxP2S12. Chem. Mater. 2018, 30 (5), 1791-1798. https://doi.org/10.1021/acs.chemmater.8b00266.

(15) Culver, S. P.; Squires, A. G.; Minafra, N.; Armstrong, C. W. F.; Krauskopf, T.; Böcher, F.; Li, C.; Morgan, B. J.; Zeier, W. G. Evidence for a Solid-Electrolyte Inductive Effect in the Superionic Conductor Li10Ge1-XSnxP2S12. J. Am. Chem. Soc. 2020, 142 (50), 21210-21219. https://doi.org/10.1021/jacs.0c10735.

(16) Dietrich, C.; Weber, D. A.; Sedlmaier, S. J.; Indris, S.; Culver, S. P.; Walter, D.; Janek, J.; Zeier, W. G. Lithium Ion Conductivity in Li2S-P2S5 Glasses-Building Units and Local Structure Evolution during the Crystallization of Superionic Conductors Li3PS4, Li7P3S11 and Li4P2S7. J. Mater. Chem. A 2017, 5 (34), 18111-18119. 
https://doi.org/10.1039/c7ta06067j.

(17) Ohno, S.; Rosenbach, C.; Dewald, G. F.; Janek, J.; Zeier, W. G. Linking Solid Electrolyte Degradation to Charge Carrier Transport in the Thiophosphate-Based Composite Cathode toward Solid-State Lithium-Sulfur Batteries. Adv. Funct. Mater. 2021, 2010620.

(18) Dewald, G. F.; Ohno, S.; Kraft, M. A.; Koerver, R.; Till, P.; Vargas-Barbosa, N. M.; Janek, J.; Zeier, W. G. Experimental Assessment of the Practical Oxidative Stability of Lithium Thiophosphate Solid Electrolytes. Chem. Mater. 2019, 31 (20), 8328-8337. https://doi.org/10.1021/acs.chemmater.9b01550.

(19) Walther, F.; Koerver, R.; Fuchs, T.; Ohno, S.; Sann, J.; Rohnke, M.; Zeier, W. G.; Janek, J. Visualization of the Interfacial Decomposition of Composite Cathodes in Argyrodite-Based All-Solid-State Batteries Using Time-of-Flight Secondary-Ion Mass Spectrometry. Chem. Mater. 2019. https://doi.org/10.1021/acs.chemmater.9b00770.

(20) Richards, W. D.; Miara, L. J.; Wang, Y.; Kim, J. C.; Ceder, G. Interface Stability in Solid-State Batteries. Chem. Mater. 2016, 28 (1), 266-273. https://doi.org/10.1021/acs.chemmater.5b04082.

(21) Zhu, Y.; He, X.; Mo, Y. Origin of Outstanding Stability in the Lithium Solid Electrolyte Materials: Insights from Thermodynamic Analyses Based on FirstPrinciples Calculations. ACS Appl. Mater. Interfaces 2015, 7 (42), 23685-23693. https://doi.org/10.1021/acsami.5b07517.

(22) Xiao, Y.; Wang, Y.; Bo, S. H.; Kim, J. C.; Miara, L. J.; Ceder, G. Understanding Interface Stability in Solid-State Batteries. Nat. Rev. Mater. 2020, 5 (2), 105-126. https://doi.org/10.1038/s41578-019-0157-5.

(23) Culver, S. P.; Koerver, R.; Zeier, W. G.; Janek, J. On the Functionality of Coatings for Cathode Active Materials in Thiophosphate-Based All-Solid-State Batteries. Adv. Energy Mater. 2019, 9 (24). https://doi.org/10.1002/aenm.201900626.

(24) Asano, T.; Sakai, A.; Ouchi, S.; Sakaida, M.; Miyazaki, A.; Hasegawa, S. Solid Halide Electrolytes with High Lithium-Ion Conductivity for Application in 4 V Class BulkType All-Solid-State Batteries. Adv. Mater. 2018, 30 (44), 1803075. https://doi.org/10.1002/adma.201803075. 
(25) Schlem, R.; Muy, S.; Prinz, N.; Banik, A.; Shao-Horn, Y.; Zobel, M.; Zeier, W. G. Mechanochemical Synthesis: A Tool to Tune Cation Site Disorder and Ionic Transport Properties of Li3MCl6 (M = Y, Er) Superionic Conductors. Adv. Energy Mater. 2020, 10, 1903719. https://doi.org/10.1002/aenm.201903719.

(26) Li, X.; Liang, J.; Yang, X.; Adair, K. R.; Wang, C.; Zhao, F.; Sun, X. Progress and Perspectives on Halide Lithium Conductors for All-Solid-State Lithium Batteries. Energy Environ. Sci. 2020, 13, 1429-1461. https://doi.org/10.1039/c9ee03828k.

(27) Hakari, T.; Fujita, Y.; Deguchi, M.; Kawasaki, Y.; Otoyama, M.; Yoneda, Y.; Sakuda, A.; Tatsumisago, M.; Hayashi, A. Solid Electrolyte with Oxidation Tolerance Provides a High- Capacity Li 2 S-Based Positive Electrode for All-Solid-State Li / S Batteries. https://doi.org/10.1002/adfm.202106174.

(28) Binninger, T.; Marcolongo, A.; Mottet, M.; Weber, V.; Laino, T. Comparison of Computational Methods for the Electrochemical Stability Window of Solid-State Electrolyte Materials. J. Mater. Chem. A 2020, 8 (3), 1347-1359. https://doi.org/10.1039/c9ta09401f.

(29) Goodenough, J. B.; Park, K. S. The Li-Ion Rechargeable Battery: A Perspective. J. Am. Chem. Soc. 2013, 135 (4), 1167-1176. https://doi.org/10.1021/ja3091438.

(30) Zhu, Y.; He, X.; Mo, Y. First Principles Study on Electrochemical and Chemical Stability of Solid Electrolyte-electrode Interfaces in All-Solid-State Li-Ion Batteries. J. Mater. Chem. A 2016, 4 (9), 3253-3266. https://doi.org/10.1039/C5TA08574H.

(31) Peljo, P.; Girault, H. H. Electrochemical Potential Window of Battery Electrolytes: The HOMO-LUMO Misconception. Energy Environ. Sci. 2018, 11 (9), 2306-2309. https://doi.org/10.1039/c8ee01286e.

(32) Tan, D. H. S.; Wu, E. A.; Nguyen, H.; Chen, Z.; Marple, M. A. T.; Doux, J. M.; Wang, X.; Yang, H.; Banerjee, A.; Meng, Y. S. Elucidating Reversible Electrochemical Redox of Li6PS5Cl Solid Electrolyte. ACS Energy Lett. 2019, 4, 2418-2427. https://doi.org/10.1021/acsenergylett.9b01693.

(33) Schwietert, T. K.; Arszelewska, V. A.; Wang, C.; Yu, C.; Vasileiadis, A.; de Klerk, N. J. J.; Hageman, J.; Hupfer, T.; Kerkamm, I.; Xu, Y.; van der Maas, E.; Kelder, E. M.; Ganapathy, S.; Wagemaker, M. Clarifying the Relationship between Redox Activity 
and Electrochemical Stability in Solid Electrolytes. Nat. Mater. 2020, 19 (4), 428-435. https://doi.org/10.1038/s41563-019-0576-0.

(34) Sendek, A. D.; Antoniuk, E. R.; Cubuk, E. D.; Ransom, B.; Francisco, B. E.; BuettnerGarrett, J.; Cui, Y.; Reed, E. J. Combining Superionic Conduction and Favorable Decomposition Products in the Crystalline Lithium-Boron-Sulfur System: A New Mechanism for Stabilizing Solid Li-Ion Electrolytes. ACS Appl. Mater. Interfaces 2020, 12 (34), 37957-37966. https://doi.org/10.1021/acsami.9b19091.

(35) Schwietert, T. K.; Vasileiadis, A.; Wagemaker, M. First-Principles Prediction of the Electrochemical Stability and Reaction Mechanisms of Solid-State Electrolytes. JACS Au 2021. https://doi.org/10.1021/jacsau.1c00228.

(36) Han, F.; Zhu, Y.; He, X.; Mo, Y.; Wang, C. Electrochemical Stability of Li10GeP2S12 and Li7La3Zr2O12 Solid Electrolytes. Adv. Energy Mater. 2016, 6 (8), 1-9. https://doi.org/10.1002/aenm.201501590.

(37) Asakura, R.; Duchêne, L.; Kühnel, R. S.; Remhof, A.; Hagemann, H.; Battaglia, C. Electrochemical Oxidative Stability of Hydroborate-Based Solid-State Electrolytes. ACS Appl. Energy Mater. 2019, 2 (9), 6924-6930. https://doi.org/10.1021/acsaem.9b01487.

(38) Ohno, S.; Koerver, R.; Dewald, G.; Rosenbach, C.; Titscher, P.; Steckermeier, D.; Kwade, A.; Janek, J.; Zeier, W. G. Observation of Chemomechanical Failure and the Influence of Cutoff Potentials in All-Solid-State Li-S Batteries. Chem. Mater. 2019, 31 (8), 2930-2940. https://doi.org/10.1021/acs.chemmater.9b00282.

(39) Dewald, G. F.; Ohno, S.; Hering, J. G. C.; Janek, J.; Zeier, W. G. Analysis of Charge Carrier Transport Toward Optimized Cathode Composites for All-Solid-State Li-S Batteries. Batter. Supercaps 2021, 4 (1), 183-194. https://doi.org/10.1002/batt.202000194.

(40) Haque, A.; Banik, A.; Varma, R. M.; Sarkar, I.; Biswas, K.; Santra, P. K. Understanding the Chemical Nature of the Buried Nanostructures in Low Thermal Conductive Sb-Doped SnTe by Variable-Energy Photoelectron Spectroscopy. J. Phys. Chem. C 2019, 123 (16), 10272-10279. https://doi.org/10.1021/acs.jpcc.9b01081.

(41) Schlueter, C.; Gloskovskii, A.; Ederer, K.; Schostak, I.; Piec, S.; Sarkar, I.; Matveyev, 
Y.; Lömker, P.; Sing, M.; Claessen, R.; Wiemann, C.; Schneider, C. M.; Medjanik, K.; Schönhense, G.; Amann, P.; Nilsson, A.; Drube, W. The New Dedicated HAXPES Beamline P22 at PETRAIII. AIP Conf. Proc. 2019, 2054 (January 2019). https://doi.org/10.1063/1.5084611. 